\title{
The IAU Strategic Plan
}

\section{George Miley}

Leiden Observatory, Leiden University, The Netherlands

\begin{abstract}
I shall review the content of the IAU Strategic Plan (SP) to use astronomy as a tool for stimulating development globally during the decade 2010 - 2020. Considerable progress has been made in its implementation since the last General Assembly.
\end{abstract}

\section{Introduction}

The IAU Strategic Plan 2010 - 2020: "Astronomy for Development: Building from IYA 2009" was ratified by the IAU General Assembly in August 2009. It is based on the unique power of astronomy as a tool for furthering human and technological capacity building throughout the world. Astronomy is a fundamental science itself and a gateway to physics, chemistry, biology and mathematics. Astronomy has been an important driver for the development of the most sophisticated technology and provides a link to our deepest cultural roots and origin.

\section{The IAU Strategy}

The Plan http://www.iau.org/static/education/strategicplan_2010-2020.pdf is a blueprint for using astronomy as a tool for development. The vision is a global one, namely that eventually all countries should participate at some level in astronomical research and that all children throughout the world will be exposed to knowledge about astronomy and the Universe. The strategy of the SP has several components:

1. A strategic phased integrated approach, including primary, secondary and tertiary education, research and public outreach. This will be based on the potential for astronomy research and education in each country, using objective data augmented by advice from experts in the region.

2. Regional involvement. A bottom-up approach, as pioneered during the International Year of Astronomy (IYA2009), involves regional input, including designation of regional institute nodes to coordinate development efforts throughout their region.

3. Special attention to Sub-Saharan Africa. Because of its relative underdevelopment, sub-Saharan Africa is receiving special attention.

4. Using IYA2009 as a springboard. Several IYA "cornerstones" are being continued and supported (e.g. UNAWE, GTTP) and the network of IYA contacts exploited.

5. Enlarging the number of active volunteers. We have recruited volunteers from amongst members, doctoral students, postdoctoral trainees, non-member experts on education and outreach and amateur astronomers. Expatriates are particularly important.

6. Exploiting innovative techniques. Innovative approaches to education and development, are being explored, including distance-learning, archives, robotic telescope networks and mobile delivery via astro-buses.

7. Creation of a small global "Office of Astronomy for Development"(OAD). Mobilising large number of volunteers, implementing new programs and inputting strategic information need professional coordination. Setting up of the IAU OAD was crucial to the success of the SP. 


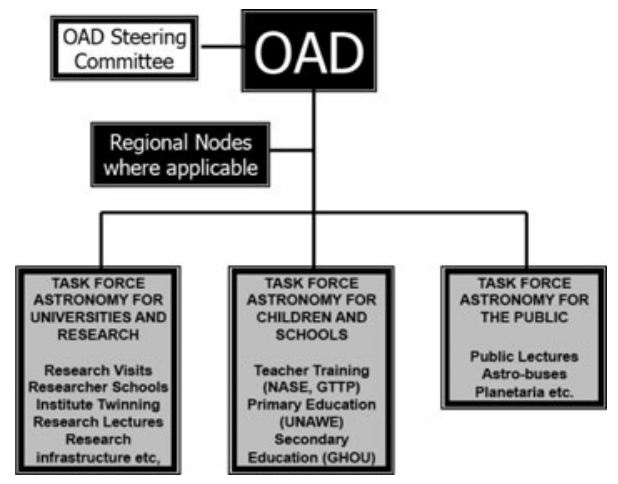

Figure 1. Structure of Astronomy for Development (AfD) activities envisaged in the IAU Strategic Plan (SP). Three task forces are coordinated by the IAU Office of Astronomy for Development (OAD) and regional input ensures a portfolio of demand-driven activities.

Figure 1 shows the implementation structure envisaged by the SP and now implemented. Three Task Forces of experts coordinated by the OAD manage the various Astronomy for Development (AfD) activities. The OAD injects a strategic component and safeguards accountability and transparency. Input from the regions ensures that the AfD portfolio matches local needs.

\section{Implementation of the IAU Plan}

The SP covers the ten-year period 2010 - 2020 and is being implemented gradually, to match the available funding. The first step was to set up the IAU Office of Astronomy for Development (OAD) to coordinate activities. Following a competitive call for proposals, and the selection of a host organisation by the IAU Executive Committee, the OAD started operation in March 2011. The IAU OAD is a joint venture between the IAU and the South African National Research Foundation (NRF), with Kevin Govender as its first Director. The official launch of the OAD took place on 16th April 2011 by the South African Minister of Science and Technology and the IAU President. By the last GA in Beijing, the OAD had set up the three Task Forces envisaged in the Strategic Plan and two regional nodes (ROADs) had been inaugurated. Enormous progress has been made in implementing the IAU Strategic Plan since then. By the end of the present GA, there will be eight functioning IAU ROADs. Earlier this year, the OAD received an oustanding report by a high-level independent review committee appointed by the IAU and the NRF. This has resulted in the intention by both the IAU and the NRF to renew the agreement between them to operate the OAD until at least 2021 and the resolution to approve this extension that we will be asked to approve later in this GA. During this period the NRF will fund an additional OAD position and the IAU will support a fund raising campaign to enable the implementation of additional activities.

During recent years it has become increasingly important for the scientific communities to justify the large expenditure on the infrastructures and facilities needed to carry out fundamental science. The IAU Strategic Plan and its implementation by the OAD is a unique endeavour for a scientific community. It demonstrates that astronomy is not only one of the most fundamental and exciting sciences, but that it can be of great benefit to society as a whole.

In the next talk Kevin Govender will next tell you more about the recent accomplishments of the OAD. 